Cahiers de recherches médiévales

Journal of medieval studies

16 | 2008

La réception d'Isidore de Séville durant le Moyen Âge tardif (XII $-\mathrm{XV}^{\mathrm{e}} \mathrm{s}$.)

\title{
Textes médicaux insérés dans les Etymologiae isidoriennes
}

\section{Carmen Codoñer}

\section{(2) OpenEdition \\ Journals}

Édition électronique

URL : https://journals.openedition.org/crm/10452

DOI : $10.4000 / \mathrm{crm} .10452$

ISSN : 1955-2424

Éditeur

Honoré Champion

Édition imprimée

Date de publication : 10 décembre 2008

Pagination : 17-37

ISSN : 1272-9752

Référence électronique

Carmen Codoñer, «Textes médicaux insérés dans les Etymologiae isidoriennes », Cahiers de recherches médiévales [En ligne], 16 | 2008, mis en ligne le 02 décembre 2008, consulté le 15 décembre 2022 URL : http://journals.openedition.org/crm/10452 ; DOI : https://doi.org/10.4000/crm.10452 


\section{酷M}

\section{Textes médicaux insérés dans les Etymologiae isidoriennes*}

Abstract: This article provides the edition and the study of a group of texts which are inserted at the end of book de medicina, after the chapter entitled de initio medicinae, in manuscripts written between the XI $I^{\text {th }}$ and XIV ${ }^{\text {th }}$ centuries. This study proves the interest of recentiores copies of the Étymologies; it also shows how this work, because of its encyclopaedic character, was continually open to supplement.

Résumé : Cet article propose l'édition et l'étude d'un groupe de textes insérés à la fin du livre de medicina, à la suite du chapitre intitulé de initio medicinae, dans des manuscrits datant du $X I^{e}$ au XIV siècle. Cette étude prouve l'intérêt des copies recentiores des Étymologies; elle montre aussi comment cette ouvre, du fait de son caractère encyclopédique, était sans cesse susceptible d'être complétée.

Il n'est pas nécessaire d'insister sur l'ampleur de la tradition manuscrite des Étymologies isidoriennes. Son énorme diffusion au cours du Moyen Âge, son statut de «manuel» indispensable dans toute bibliothèque, transforment l'étude de la tradition manuscrite en un problème interminable. Dans ce cas, cette tâche est compliquée par l'existence d'indices qui incitent à postuler l'existence de plusieurs rédactions.

En se basant sur l'étude de quelques-uns des très nombreux manuscrits existants, on a proposé plusieurs hypothèses pour expliquer les phases de la transmission depuis le moment où le texte est sorti (ou, si nous admettons plus d'une « édition », les textes sont sortis) pour la dernière fois des mains d'Isidore. Ces travaux, depuis Lindsay, ont abouti à reconnaître une transmission en trois familles, dont le dernier stemma fut dessiné par Reydellet ${ }^{1}$. À partir de cette base a vu le jour le projet d'édition des Étymologies : vingt éditeurs devaient se charger des vingt livres et la solution définitive à chacun des problèmes posés, si tant est que la notion de «définitif» soit admissible dans ce type de travaux, devait émerger de la somme d'informations issues de l'édition de chacun des vingt livres qui composent les Étymologies. Cette masse de données devait offrir une vision multiple et en même

\footnotetext{
* Je remercie Jacques Elfassi pour avoir traduit en français cet article et révisé l'apparat critique. Je remercie aussi K. Fischer pour avoir révisé le texte latin; le texte a pu être amélioré grâce à cette révision.

${ }^{1}$ M. Reydellet, «La diffusion des Origines d'Isidore de Séville au Haut Moyen Âge», Mélanges d'Archéologie et d'Histoire de l'École Française de Rome, 78, 1966, p. 383-437. Bien que je ne sois pas entièrement d'accord avec lui, je ne suis parvenue à aucune conclusion fondée sur des arguments suffisamment solides pour proposer un nouveau stemma offrant les meilleures garanties. C'est pour cette raison que j'ai décidé de le garder dans la partie consacrée aux Etymologiae dans P. Chiesa et L. Castaldi (éd.), La trasmissione dei testi latini del Medioevo. Mediaeval Latin Texts and their Transmission. Te.Tra. 2, Firenze, SismelEdizioni del Galluzzo (Millennio medievale, 57 ; Strumenti e studi, n.s. 10), 2005.
}

Cahiers de Recherches Médiévales, 16, 2008 
temps plus nette. En effet, le projet incluait aussi l'élaboration d'un volume introductif qui devait tirer les conséquences du travail individuel mené à bien par chaque éditeur.

Malheureusement, les éditeurs des différents livres ont vu leur tâche trop facilitée. L'extraordinaire travail de Reydellet réduisait les possibilités d'apporter quelque chose de nouveau : les manuscrits soigneusement sélectionnés avaient déjà été analysés de différents points de vue, le stemma était établi et l'existence de la contamination mise en évidence. L'unique contribution possible, si on exclut l'addition d'un commentaire, était limitée à une collation adéquate des manuscrits recommandés qui, parce qu'il s'agit de livres isolés, ne pouvait modifier en profondeur la vision que l'on avait déjà de l'ensemble. En théorie l'entreprise était irréprochable. Toutefois, il est utopique de penser qu'un éditeur va se limiter à «son» livre quand celui-ci ne présente pas un caractère clairement indépendant, c'est-à-dire si le livre à éditer acquiert seulement sa vraie spécificité comme partie à l'intérieur du tout - c'est le cas de plusieurs livres. Il est possible qu'à la fin il parvienne aux mêmes conclusions que celles qui ont déjà été obtenues par Reydellet, mais les problèmes ne doivent pas être éludés.

L'étude des travaux existants sur la transmission de l'œuvre me mit en contact avec de nouveaux manuscrits qui, à leur tour, m'amenèrent à d'autres. Leur connaissance directe, sans que je me circonscrivisse au livre X, me permit d'ajouter de nouveaux éléments aux manuscrits déjà connus; et finalement, comme cela arrive toujours, pour essayer de trouver une explication aux questions non résolues, je m'avançai vers les recentiores. Ce travail est une petite contribution à la connaissance d'une branche de la tradition présente dans plusieurs manuscrits du $\mathrm{XII}^{\mathrm{e}}$ siècle ou du début du XIII $\mathrm{e}^{\mathrm{e}}$ siècle; il s'agit des copies suivantes: Milano, Ambrosiano H 187; BAV Ottob. 477 et Lat. 623 ; El Escorial b.I.12; R.II.11 et \&.I.12 ; Paris, Mazarine 689 ; et Toulouse, Bibliothèque Municipale 176.

Tous ces manuscrits ont intercalé dans le texte des Étymologies isidoriennes une série d'additions de taille et de contenu variés².

Ce qui m'intéresse ici est un texte inséré à la fin du livre de medicina, à la suite du chapitre intitulé de initio medicinae $e^{3}$. L'ensemble de l'édition est constituée par plusieurs pièces dont l'origine est peut-être différente.

Dans un article de $2005^{4}$ j'ai tenté de localiser l'origine possible de l'introduction par laquelle commence la première pièce. L'existence d'une introduction semblable au Livre d'Asaph ${ }^{5}$, un Initium medicine édité par Sudhoff et

\footnotetext{
2 J'ai l'intention de consacrer un travail approfondi à l'étude des additions considérées dans leur ensemble, mais en détaillant chacune d'elles.

${ }^{3}$ Dans El Escorial R.II.11 il est situé entre les chapitres 12 et 13 du livre de medicina.

${ }^{4}$ «La medicina en algunos manuscritos de Isidoro de Sevilla», Isidorus medicus. Isidoro de Sevilla y los textos de medicina, éd. A. Ferraces Rodríguez, La Coruña, Universidade da Coruña, 2005, p. 65-84

${ }^{5}$ A. Jellinek, Bet ha-Midrash: Sammlung kleiner Midrashim und Vermischter Abhandlungen aus dem alteren judische Literatur, vol. 3, Leipzig, C. W. Vollrath, 1855, p. 156. Voir E. Lieber, "Asaf"s book of Medicines : a hebrew Encyclopedia of greek and jewish medicine, possibly compiled in Byzantium on an Indian model», Dumberton Oak Papers, 38, Symposium on Byzantine Medicine, 1984, p. 233-249.
} 
une légende hébraïque de même contenu ${ }^{6}$, m'ont amenée à penser à plusieurs adaptations d'un texto hébreu commun?

Dans la suite de ce qui vient d'être dit, le texte que nous étudions ici est la somme de plusieurs «histoires de la médecine» suivies d'une brève anatomie. S'y ajoutent ensuite deux brefs traités, déjà édités dans la première moitié du $\mathrm{XX}^{\mathrm{e}} \mathrm{s}$. et dont je ne vais pas m'occuper ${ }^{8}$.

Toutes ces «histoires de la médecine» ont été considérées comme une continuation du livre IV De medicina de l'évêque de Séville. Par conséquent, elles reçoivent une numération qui part du chapitre final (XIII) du livre IV ; et donc, bien que la numérotation s'adapte à une distribution différente des parties selon les manuscrits, elle part toujours du chapitre XIII comme référent numérique.

Voici comment $j$ 'analyse les pièces qui composent cette addition :

(A) Une première partie, commençant par l'introduction au Livre de Noé, attribue l'origine de la médecine aux enseignements de l'archange Raphaël à Noé, qui transmet son savoir à son fils Sem. Des sages de diverses régions continuent la recherche. Après un intervalle de centaines d'années, l'effort pour chercher des remèdes aux maladies s'incarne en Asclépios. C'est la partie qui coïncide avec le début du traité d'Asaph l'Hébreu'.

On pourrait peut-être voir dans la fin de cette section une réélaboration du texte introductif au livre d'Asaph. En effet, il s'agit d'un paragraphe (on ne peut même pas parler de section) qui correspond approximativement à la phrase initiale d'un ars medicinae édité par Laux, parlant des origines d'Asclépios ${ }^{10}$. S'y ajoute une phrase finale qui inclut la figure d'Asaph dans la liste traditionnelle des pionniers de la médecine : Hippocrate, Asaph, Discorius (sic) et Galien.

${ }^{6} \mathrm{~K}$. Sudhoff, «Ein neuer text der Initia medicine », Mitteilungen zur Geschichte der Medizin und der Naturwissenschaften, 15, 1916, p. 281-287; S. Muntner, «The antiquity of Asaph the Physician and his editorship of the earliest Hebrew book of medicine ", Bulletin of the History of Medicine, 15, 1951, p. 101-131.

${ }^{7}$ Dans l'article, je n'exclus pas l'hypothèse de la dépendance du texte de Sudhoff par rapport à la version latine. Une intervention de Vivian Nutton sur un texte voisin dans un manuscrit de la Bibliothèque Vaticane (Vat. lat. 2378) m'a fait comprendre que cette hypothèse était peu vraisemblable; en effet, les textes hébreux comportent tous un élément qui ne se trouve pas dans la version latine : la permission de la part de Dieu de laisser libre un des esprits du Mal pour qu'il châtie les hommes mauvais. Je remercie V. Nutton pour m'avoir permis de lire son article avant sa publication.

${ }^{8}$ M. Wlaschky, «Sapientia artis medicinae. Ein frühmittelalterliches Kompendium der Medizin », Kyklos, 1, 1928, p. 103-113. Aux manuscrits cités par l'auteur on peut ajouter le ms. 88 de la Bibliothèque Municipale de Vendôme, selon ce qui ressort de la description de H. E. Sigerist, «Early medieval medical texts in manuscripts of Vendôme », Bulletin of the History of Medecine, 14, 1943, p. 68-113. Le titre est le même, la partie qui nous intéresse occupe les $138^{\mathrm{v}}-139^{\mathrm{v}}$; l'incipit et l'explicit de cette partie est aussi la même. Le manuscrit de Vendôme continue avec la partie III de la Sapientia...

${ }^{9}$ Voir note 16.

${ }^{10}$ R. Laux, «Ars medicinae. Ein frühmittelalterliches Kompendium der Medizin », Kyklos, 3, 1930, p. 417-434, qui coïncide avec notre texte seulement sur l'ascendance d'Hippocrate. 
Ce paragraphe pourrait être interprété comme un colophon à l'exposé précédent ou bien comme une espèce de transition à la partie doctrinale, laissant une place à la tradition profane.

La partie du chapitre présent dans Laux (quoique avec des variantes) coïncide partiellement avec le chapitre 3 du livre de medicina d'Isidore. Il est intéressant de voir les divergences entre le récit proposé par notre texte et celui d'Isidore :

Addition
Tunc et medicina simul cum
auctoribus defecit latuitque
per annos pene quingentos
triginta usque ad tempus
Artaxersis regis Persarum. Tunc
eam reuocauit in lucem Ypocrates,
patre Asclepio natus in insula
Choo,
et alii sapientes gentium, id est
Asaph Hebreus, Discorius
Balatheus et Galienus
Cappadocenus et multi alii
sapientes reliqui renouauerunt
artem medicine que permanet usque
in odiernum diem.

ISIDORE (éd. Lindsay)

Et ars simul cum a uctore (sc. Aesculapius) defecit, latuitque per annos pene quingentos usque ad tempus Artaxersis regis Persarum. Tunc eam reuocauit in lucem Hippocrates Asclepio patre ge n it us in insula Coo.

Il est manifeste que le texte a été adapté, sans préjuger de l'antériorité de l'une ou l'autre version : un lien est établi avec le développement qui précède. La simple transformation d'auctore en auctoribus, ou vice versa, suffit à le montrer; et si elle ne suffisait pas il y a la partie finale du passage. La précision triginta est un autre petit détail qui les distingue.

C'est cette seconde partie de la pièce qui a servi à dater l'époque où a vécu Asaph l'Hébreu ${ }^{11}$.

À partir de là commence la partie doctrinale, exposant une doctrine attribuée à Asaph, comme l'indiquent plusieurs intitulés du bref traité. Il comprend une brève information sur les humeurs, les os et une description plus étendue sur les veines.

Ensuite, et attribué explicitement à Asaph, un chapitre très long De partibus corporis. Suivent De quattuor humoribus et De partitione anni in quattuor circulis.

${ }^{11}$ L. Ginzberg, dans The legends of Jews (tr. Henrietta Szold), London-Baltimore, Johns Hopkins University Press, 1998 (= Philadelphia, Jewish Publication Society of America, 1909), vol. I, cap. 4, rapporte la légende, mais dans ce cas il change l'ordre et place Asaph à la fin de la série. Ce déplacement d'Asaph à une autre position à l'intérieur du texte légendaire, tel que l'offre Ginzberg, affaiblit la datation du personnage proposée par des érudits antérieurs. Effectivement, il passerait du $\mathrm{I}^{\mathrm{er}}$ s. av. J.-C. ou $\mathrm{I}^{\mathrm{er}}$ s. ap. J.-C. à une date postérieure à Galien, sans plus de précisions. Voir E. Lieber, art. cit. Il est curieux, toutefois, que le même Ginzberg, dans son article sur Asaph, dans The Jewish Encyclopedy, écrit en 1901, suive l'ordre du Livre de Noé pour le dater. 
La présence dans la partie suivante, De natura cerebri secundum Asaph, d'une phrase initiale Aitque Asaph... et un explicit qui consiste en une sententia attribuée aussi à Asaph, confirment à mon avis que tous les chapitres antérieurs aussi pourraient lui être attribués. Avec ce chapitre se clôt cette première pièce médicale, dont l'origine pourrait être hébraïque.

(B) Puis commence un deuxième exposé, cette fois sans aucune attribution. Comme dans le cas précédent il commence par une version, sous forme narrative, des origines de la médecine, dans ce cas de fondement grec : l'hippocentaure qui transmet ses connaissances à Hippocrate, qui, à son tour, dans un dialogue de maître à disciple, les transmet à Galien.

(C) Nous passons à une troisième partie, de contenu nettement chrétien, introduite comme les précédentes par un bref passage sur les «inventeurs de la médecine », dans ce cas Asclepios et Apollon, qui transmettent leurs connaissances à Hippocrate.

Son premier chapitre, De assimilatione elementorum, met en relation les quatre éléments avec quatre aspects de la doctrine chrétienne. La transformation de terra et aer en puluis et uentus est intéressante : ainsi, l'assimilation du feu au péché et de l'eau à la charité est suivie de l'assimilation de puluis à terra et de uentus à aer, rapprochement qui est clairement d'ascendance biblique. Cette introduction sert de base à la conclusion finale : sicut de quatuor causis constat corpus humanum, ita mundus similiter de quatuor causis, et à la conclusion de cette section consistant en la division du corps en quatre parties: Corpus humanum in quatuor partibus diuiditur... qui continue les correspondances entre le chiffre quatre et la création, bien qu'en développant la comparaison elle se limite à la tête (caput est quarta pars cerebri...), la première des parties citées.

Ensuite nous trouvons un paragraphe un peu surprenant, parce qu'il est partiellement une répétition (avec un changement) du dernier passage que nous avons vu, c'est-à-dire la division du corps humain. Il est basé sur la définition de toutes les parties de l'être humain qui correspondent aux chiffres quatre et cinq. La rédaction est quelque peu maladroite :

Quinque talenta sunt in natura humana... et in quatuor partes diuiditur corpus humanum...

Quatuor humores sunt in humano corpore...

Il s'agit d'une seconde définition des quatre parties du corps, mais cette fois pectus est remplacé par stomachus et umbilicus par uenter. À chacune des parties est assignée une fonction. Après l'énumération des quatre humores le texte reprend le motif des parties du corps, mais en faisant allusion à une seule d'entre elles, le pectus, qui avait été remplacé par stomachus, comme je viens de le dire. Il en donne une définition qui contredit sa condition de pars corporis, puisqu'il dit qu'il occupe la partie supérieure de l'estomac, qui avait été inclus comme une des parties du corps.

Le caractère hétérogène de ce bref fragment, qui répète les parties du corps humain, déjà mentionnées, qui introduit l'idée des contraires déjà introduite à la fin de la partie précédente et qui énumère les quatre humeurs - énumération qu'on 
trouve à nouveau dans la section suivante-, fait penser que nous sommes devant un fragment indépendant, avec une entité propre.

C'est seulement à partir de cet endroit que commencent les coïncidences avec le texte édité par Wlaschky au début du $\mathrm{XX}^{\mathrm{e}}$ siècle $^{12}$ : Quatuor humores sunt in humano corpore...

Le fait que ce soit à cet endroit que commence le texte I du groupe de quatre publiés sous le nom d'Initia medicinae, accrédite l'idée que le paragraphe débutant par quinque talenta est un fragment qui sépare le développement de contenu chrétien de la partie consacrée à la médecine proprement dite.

Ici commence, donc, la partie correspondant aux deux premières sections du Sapientia artis medicinae. Une différence légère, mais significative, les sépare. Le texte I d'Initia a un paragraphe initial qui n'existe pas dans le texte transmis par nos manuscrits :

Quattuor sunt uenti, quattuor sunt anguli caeli, quattuor tempora: uernum, aestiuum, autumnus et hiems.

À partir de là le texte, avec les variantes qui sont la conséquence d'une transmission distincte, est identique ${ }^{13}$.

Nous allons maintenant éditer les parties antérieures au texte Sapientia déjà édité. Il paraît évident que la partie initiale connaissait une existence antérieure indépendante, bien que nous n'en connaissions pas le modèle.

Ce que nous pouvons attribuer à l'auteur de l'ensemble d'une insertion aussi longue, c'est la somme des diverses pièces, l'adaptation de la seconde de ses pièces à ses propres critères et peut-être l'élaboration (qu'elle lui soit propre ou qu'il l'ait empruntée) des éléments unissant les deux pièces. Les Etymologies étaient considérées comme un vrai compendium des connaissances nécessaires, ce qui faisait de cette « encyclopédie » le lieu adéquat pour conserver de nouvelles idées ou des doctrines complémentaires sur toutes les matières, en ce cas précis la médecine.

\section{Édition}

Comme il s'agit d'un texte dont la tradition est indépendante du texte de base dans lequel il a été inséré, les caractéristiques générales des manuscrits n’apportent

${ }_{12}$ M. Wlaschky, «Sapientia artis medicinae. Ein frühmittelalterliches Kompendium der Medizin », Kyklos, 1, 1928, p. 103-113. Aux manuscrits cités par l'auteur on peut ajouter le ms. 88 de la Bibliothèque Municipale de Vendôme, selon ce qui ressort de la description de H. E. Sigerist, "Early medieval medical texts in manuscripts of Vendôme », Bulletin of the History of Medecine, 14, 1943, p. 68-113. Le titre est le même, la partie qui nous intéresse occupe les $138^{\mathrm{V}}-139^{\mathrm{v}}$; l'incipit et l'explicit de cette partie est aussi la même. Le manuscrit de Vendôme continue avec la partie III de la Sapientia...

${ }^{13}$ Ce texte apparaît sous le titre d'Epistola Isidori dans le ms. Vat. lat. 4418 de la Biblioteca Apostolica Vaticana. 
pas grand-chose à l'analyse du texte édité. C'est pourquoi je me limiterai à fournir les informations essentielles sur les manuscrits que j'ai utilisés et à proposer un regroupement schématique par familles.

Pour ce qui est des normes suivies, j'ai limité l'apparat critique aux variantes significatives, en négligeant les variantes orthographiques. J'ai normalisé les graphies dans tous les cas où il n'y a pas accord des manuscrits.

Transmission manuscrite ${ }^{14}$ :

$A$ Biblioteca Ambrosiana H 187inf.

Parch., XI s., cc. I + 231 + I, 300 x 210 mm (c. 1), le folio 1 manque. fol. $83^{\mathrm{v}}$ (olim $\left.84^{\mathrm{v}}\right)$ Item aliter de medicina...

fol. $88^{\mathrm{r}}\left(\right.$ olim $\left.89^{\mathrm{r}}\right) \ldots$ sanus efficitur. explicit liber v (v deletus)

$B$ BAV Ottob. Lat. 477

Parch., XIII s., 2 col.

f. $44^{\text {ra }}$ De initio medicine...

f. $48^{\text {rb }} \ldots$ sanus efficietur.

$T$ Toulouse, Bibliothèque Municipale 176

Parch., fin du XIII ${ }^{\mathrm{e}}$ s., 274 ff., 2 col.

f. $58^{\text {ra }}$ In diebus denique illis...

f. $63^{\text {ra }} \ldots$ et sanus efficitur.

$V$ BAV Vat. Lat. 623

Parch., XIII-XIV ${ }^{\mathrm{e}}$ s., Italie, I-IV + 156 ff., 315 x 224 mm., 2 col. fol. $39^{\text {rb }}$ de causa et exordio...

$42^{\text {ra }} \ldots$ et sanus efficitur.

$C$ El Escorial b.I.12

Parch., XIV ${ }^{\mathrm{e}}$ s., 216 ff., 295 x 215 mm., 2 col. Manuscrit très corrigé.

f. $54^{\mathrm{r}}$ de causa et exordio medicine... xiiii

f. $58^{\mathrm{r}} \ldots$ et sanus efficietur.

$M$ Paris, Bibliothèque Mazarine 689

Parch., XIV s., 235 ff., 320 x 230 mm., 2 col. À la fin du ms., main du XVI s. : « Pro libraria regalis collegii Navarre, de dono M. Alexandri, quondam janitoris ejusdem $»$.

f. $60^{\text {ra }}$ de causa et exordio medicine...

f. $64^{\mathrm{vb}} \ldots$ et sanus efficietur.

$R$ El Escorial R.II.11

Parch., XIV ${ }^{\mathrm{e}}$ s., 249 ff., 298 x $118 \mathrm{~mm}, 2$ col.

f. $46^{\text {ra }}$ de ignitio [sic] medicine... 14 .

f. $49^{\text {ra }} \ldots$ et sanus efficitur.

\footnotetext{
${ }^{14}$ J'ai collationé aussi El Escorial \&.I.2, mais il coincide presque totalement avec $V$, de sorte que je le cite seulement quand il s'en éloigne.
} 
$A$ est le manuscrit le plus ancien et aussi le plus fiable. Bien que les liens de parenté dans la transmission des Etymologiae ne soient pas décisifs dans l'étude de cette addition, il est intéressant de constater qu'il a comme point commun avec $K$ (Wolffen. 64) et $C$ (Leiden F. 74) l'omission de toutes les lettres sauf la dernière, celle dans laquelle Isidore annonce qu'il a envoyé à Braulion les Étymologies, la double dédicace des livres (à Braulion et Sisebut) : En uobis, et l'absence du Vt ualeas $^{15}$.

Dans la transmission du texte que nous éditons nous avons deux manuscrits clairement différenciés qui sont à la tête de deux branches : $A$ et $V$. Le manuscrit $T$ dépend de $A$, avec qui il partage de multiples omissions et additions.

Par ailleurs, on peut considérer $B C M R$ comme un groupe d'origine commune, bien que les influences et les interférences dans l'une ou l'autre direction soient nombreuses.

Quant à la complexité des relations entre l'ensemble des manuscrits, il suffira de proposer deux exemples pour en donner une idée. Le premier se trouve à la 1.66 :

forcia uirtutis hominis $A$

forcia uirtus uel uirtutis $h . R V T$

forma uirens $h . m g$. al. forcia uirtus $B$

forma uirens uel uirtutis $h$. $C M$

Nous pouvons reconstruire le processus de la manière suivante. Le texte de l'archétype avait: fortia hominis. Marginalement ou entre les lignes s'est ajouté comme glose à fortia un uirtus destiné à l'expliquer, glose qui s'intègre au texte : fortia uirtus hominis. D'un côté, $A$ n'a pas compris l'expression et l'a corrigée en forcia uirtutis. De l'autre, $R V T$, peut-être sous l'influence de deux modèles, retrouvent l'original forcia uirtus, mais en donnant la glose comme alternative : uirtutis. Enfin, un groupe de trois copies $(B C M)$ lit de manière erronée forma uirens, mais ils se séparent dans l'option choisie comme alternative: forcia uirtus ou uirtutis.

Autre exemple (1. 222) :

artem medicine $A$

artem medicinalis scientie $T$

artem medicinalem scientie $V$

artem medecinalem/medinalem $R C$

La leçon de l'archétype était peut-être medicine. À partir de là a eu lieu une série de développements qui ont peut-être comme point de départ la transformation du substantif au génitif medicine en un adjectif medicinalem, ce qui peut expliquer ensuite le reste des versions.

Devant une situation semblable, nous avons jugé préférable de ne pas établir de stemma; nous nous limitons à accepter l'existence de deux branches,

\footnotetext{
${ }^{15}$ Sur toutes ces questions, on peut consulter C. Codoñer, «Isidorus Hispalensis ep. 1. Etymologiae », La trasmissione dei testi latini del Medioevo. Mediaeval Latin Texts and their Transmission. Te.Tra. 2, P. Chiesa et L. Castaldi (éd.), Firenze, Sismel-Edizioni del Galluzzo, 2005 (Millennio Medievale, 57 ; Strumenti e Studi, n.s. 10), p. 274-299.
} 
représentées par $A$ et $V$, et à souligner l'affinité de $T$ avec $A$ et celle de $B C M R$ avec $V$, soit dans leur ensemble soit séparément.

Carmen Codoñer Université de Salamanque 


\section{A. DE CAVSA ET EXORDIO MEDICINE ET A QVIBVS HABVIT INITIVM ET NOMEN}

In diebus denique illis et in tempore illo, quia multis erat mundus repletus sceleribus, ut impleretur quod dictum est in Libro Sapientiae, pugnauit pro deo orbis terrarum contra insensatos. Ideo apostate angeli ad uindictam criminum uarios morbos ac diuersas infirmitates humano generi tulerunt, et disciplinam piissimi patris per cataclismum exhibitam superbia humana suscipere renuit, suis exigentibus meritis ut malignis spiritibus miseri homines deludendi traderentur. Preterea ueniens omnis illa generatio ad Noe patrem suum narrauit ei quibus et qualibus generibus mortium affligerentur. Tunc conturbatus est ualde Noe cognouitque per sue prudentie spiritum quia pro innumeris piaculis hec humanum genus plaga uastaret.

Sanctificatis itaque Noe ab omni inmunditia filiis et filiabus suis omnique domo sua accessit ad altare quod ab eodem post egressionem fuerat edificatum arce totisque uiribus deprecatus dominum ut hanc pestem sua pietate deleret. Misertus itaque dominus et condolens miseriis hominum, misit unum ex vii angelis stantibus ante se, cui nomen Raphael, quod est interpretatum medicina Dei ut, omnes inmundos spiritus ac potestates aereas sua uirtute cohercens ultra humanum genus ab huiusmodi plaga non deleretur.

Veniens itaque angelus, ut aiunt, ostendit omnem in arboribus et herbis uariisque pigmentis medicinam deditque ad Noe. Deinde Noe, collectis in unum nominibus arborum herbarumque $\dagger$ radicibus uarios foliorum flores $\dagger$ ac diuersi generis pigmenta uolumen conscripsit et tradidit Sem filio suo.

Et abinde, ut ferunt, nomen medicine uidetur exortum. Postea uero prudentes, primo de isto libro transtulerunt et scripserunt unusquisque secundum linguam gentis sue et multiplicata est scientia medicine super faciem terre per uarias terrarum

1 Item aliter de medicina quomodo primitus inuenta fuit xiii (capit. xiiiim $E$ ) $A E$ de initio (ign- $R$ ) medicine et quom et a quibus habuit initium $\&$ nomen xiiii $B R$ xiiii $C$ de causa et exordio medicine et a quibus habuit initium et nomen $M$ hec est additio usque in finem quarti libri $m g$. add. $E$ sine titulo $T \quad 3$ pugnabit $E \quad$ deo] eo $E \quad$ omnis $B R \quad \mathbf{4}$ terra $R$ insensatos --- ad om. $T$ insensatatos postea corr. $C$ ideo] id est $R$ apostate] a potestate $M$ uindicatam $C$ iustam $T$ uarios] omnes B $\mathbf{5}$ intulerunt $C T$ et scripsi] ut codd. 6 catha- $C R T$ superbia humana scripsi] superbiam humanam codd. renuit $V$ rennuit $A$ tenuit $B C R \quad 7$ meritis ut scripsi] meritis codd. misereri $A$ deludendi] ne ludendi $A$ 8 omnes $R \quad$ ad noe ante omnis colloc. $T$ narrauerunt $M T V$ eis $R$ qualibet $M$ 8/9 generibus mortium transp. $B T \quad 9$ mortui $R \quad$ aflig- $R$ affligeretur $A$ tun $V$ 10 quia] -que $R \quad$ pro] per $T$ humanum genus transp. $B R \quad$ plagam $R$ uastaret $A T$ ] mon(mos- B)straret BCMR monstraret postea corr. $V \quad \mathbf{1 1}$ noe om. AR et post. add. $C$

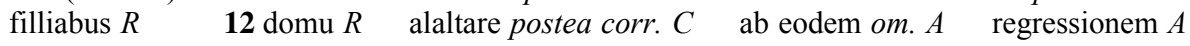
arce] arce $A \quad \mathbf{1 3}$ totis $C \quad$ deprecatur $M(m g$. add.) $T \quad \mathbf{1 4}$ itaque dominus om. $T$ 16 aerias $A V T \quad \mathbf{1 7}$ huius $C$ non iter. $V$ deleretur $B C R T]$ lederetur $A$ lederet $V$ 18 angeli $T \quad 19$ ad om. $B C R \quad 20$ filiorum $R \quad 21$ uolumen conscripsit transp. $A$ $\mathbf{2 2}$ abinde ut ferunt] alii fuerunt (inde ut ferunt $m g$.) $C$ fertur $R$ medecine $R$ 23 primo] primis $A$ isto om. $C$ terustulerunt $T$ scripsit $A$ 
nationes. Non sunt itaque inuenti sapientes ex omnibus gentibus qui sic inuestigarent et cognoscerent huius artis peritiam sicut sapientes Indie, Syrie et Egyptiorum et Macedoniorum. Sapientes itaque Indie perscrutati sunt omnem scientiam in lignis et pigmentis.

Sapientes uero Syrie inuenerunt scientiam in herbis et seminibus terre. Prudentes uero Macedonie uidentur primum in huius artis peritia claruisse. Sapientes uero Egiptiorum ceperunt auguriari secundum suam scientiam in astris celi et in uariis incantationibus ; et ipsi inuenerunt librum Chaldeum, quem transtulit Cinarius filius Hur, filii Cedebe, qui fuit auctor auruspicum et incantationum.

Creuit itaque horum scientia in libris medicinalibus ualde, donec surgeret Asclepius, unus ex Macedonum sapientibus et quadraginta uiri cum eo auruspices docti et perambulantes Indiam uenerunt in terram Nahor, qui est locus proximus Paradiso, ut inquirerent lignum uite et magnificarent gloriam suam super omnes sapientes terrarum. Cum uenissent ad locum illum inuenerunt uaria artis medicine ligna. Sed et lignum uite contra fas inquirere cupientes, uersatilis gladius qui ob angelorum custodiam malorum ante Paradisum collocatus est, eos percutiens flammarum ictibus interfecit et nec unus quidem ex eis remansit.

Tunc et medicina simul cum auctoribus defecit latuitque per annos pene sexcentos xxx usque ad tempus Artaxersis regis Persarum. Tunc eam reuocauit ad lucem Ypocrates, patre Asclepio natus in insula Choo, et alii sapientes gentium, id est, Asaph Hebreus, Discorius Balatheus et Galienus Cappadocenus et multi alii sapientes reliqui renouauerunt artem medicine que permanet usque in hodiernum diem.

Ipsi inuestigauerunt et exquisierunt omnem compaginem corporis hominum. Quomodo ex quatuor humoribus corpora consistunt hominum id est, ex humido et sicco et calido et frigido. Quia sicut ex quatuor elementis consistit mundus, ita ex

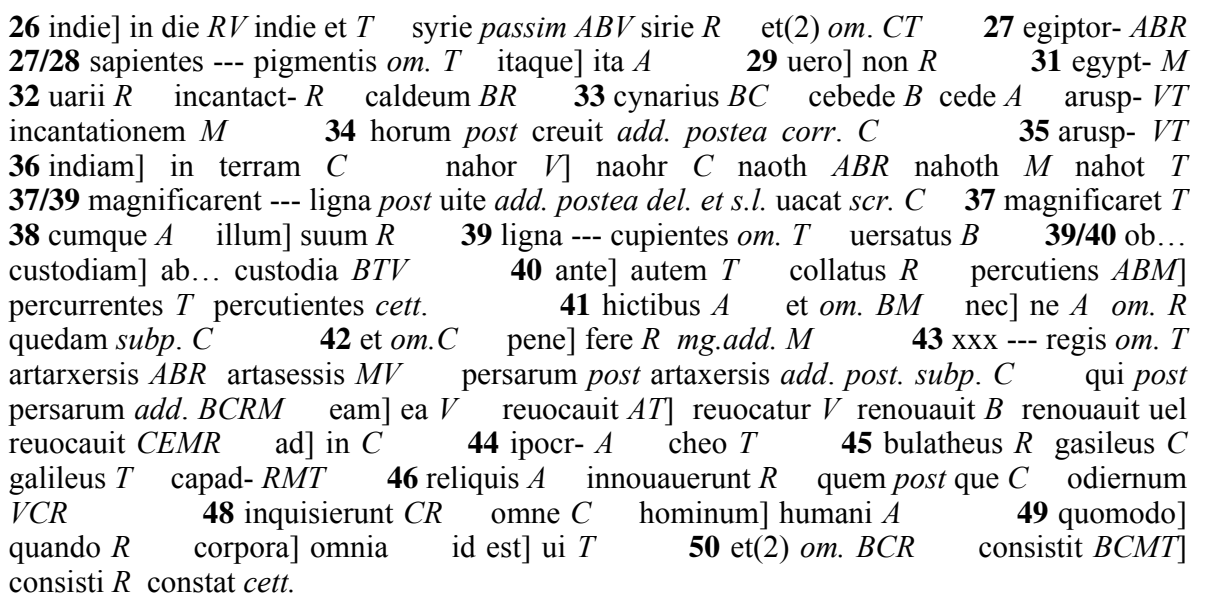


quatuor humoribus constat homo. Mundus iste constat ex celo et terra ex aqua et igne ; et homo ex sanguine et colera, ex flegmate et melanconia.

In corporibus itaque hominum sex generum sapores consistunt atque inter se repugnare uidentur, id est, dulcis, acidus, amarus et salsus, acutus et sapor galle ; et effigies personarum hominum in xii partibus terminatur, et ducentis xlviii artubus diuiditur et cccxl uenis homo distensus est. Ipse sunt aqueductus que detrahunt sanguinem ad pergendum per totum corpus hominum; et tote iste distense et suspense sunt in quinque uirtutibus que sunt in corpore hominum.

Hec sunt diuisiones ossuum per membra sua. Incipiunt a planta pedis ossa tria, et per omnem digitum ossa $\mathrm{v}$ et super planta pedis ossa quinque et per omne genu ossa tria, et duo ossa in tibia et in cossa unum, et super nates quod est cudurile os unum et iacentia illo et illo lumbo ossa viii que sunt duplicia et faciunt xvi ; et tria ossa in interiora, et xviii nodora spinalia. Et ad dexteram partem hominis coste ix et in sinistra parte hominis coste viii, que coniunguntur in ipso spinali. Et in scapula una ossa vi, et in scapula secunda similiter vi. Et quinque ossa sunt ad concludendum cor, et ibi est forcia hominis. Et in cerebro eius ossa ix et viii in pectore. Et per omnes artus manus ossa $\mathrm{v}$ et per uniuersos digitos manuum ossa $\mathrm{v}$.

Ista sunt ossa hominum per omnia membra eius absque dentibus et aliis ossibus minutis; et uene sunt que distenduntur per omnia membra hominis ad irrigandum corpus ad uiuacitatem anime per sanguinem ad omnem carnem illius. Quomodo ergo diuiduntur omnes uene per uniuersum corpus explanemus.

Est uena principalis in dextera parte ceruicis et ab ea exeunt xvi uene que expandunt se ad interiora iecoris. Alie uero vii pertrahunt se in pectore.

Similiter alie iiiior pergunt in cerebro capitis et due sunt maiores que extendunt se una a dextro et altera a sinistro usque ad ceruicem. Ex ipsis duabus

51 constat] consistit $M \quad \mathbf{5 2} \operatorname{ex}(1) A V T]$ et cett. $\quad \operatorname{ex}(2)]$ et $M \quad$ melanconia $A V C$ (postea corr.C)] melancolia $B R M$ melenconia $T \quad 53$ in --- consistunt om. $T$ existunt $R$ $\mathbf{5 4}$ acidus amarus et salsus] acidum amarum salsum $A T V \quad \mathbf{5 5}$ terminantur $T$ terminat $R$ $248 R \quad 56$ et iter. $T \quad$ cccxl] $\operatorname{ccclx} A B T$ ccc.9. $R \quad$ ipse] iste $B V$ quo $T$ trahunt $R$ 57 distente $A$ distincte $C \quad 58$ in --- sunt om. $T$ quinque] $.5 . R$ corpore $A C V$ 59 ossuum $A$ ] ossium cett. pedis] pedum $C \quad \mathbf{6 0}$ et super --- quinque om. $B C R M$

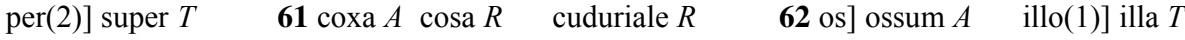
lumborum coniec. Fischer que] quod $A V$ sunt + super post.corr. $C$ duplicia] duplices $A$ duplicata $C \quad \mathbf{6 3}$ in om. $C$ (postea add. s.l.) $M \quad$ xviii $A \quad$ nodorum $B$ $\begin{array}{llll}\text { spinalis } A & \text { dextram } V & \mathbf{6 4} \text { hominis } \text { om. } A & \text { coniunguntur } C \text { ] coniungunt se } A\end{array}$ coniungunt $B V \quad$ spinale $A \quad \mathbf{6 5}$ in om. $T$ et in scapula secunda similiter vi post vi add. postea subp. $C \quad$ ad] in $T \quad \mathbf{6 6}$ forcia scripsi] forcia uirtutis $A$ forma uirens $(m g$. ał. forcia uirtus) $B$ forcia uirtus uel uirtutis $R V T$ forma uirens uel uirtutis $C M$ eius $A V$ ] hominis $B C E M R \quad 67$ et $\operatorname{per}(2) \ldots$ ossa om. $R \quad \operatorname{per}(2)$ om. $E C \quad$ uniuersa digita $A$ manuum $A$ ] manum $T$ manus cett. $\quad 68$ ossa om. $T \quad 69$ ossibus minutis transp. BCMR sunt $o m$. $A R \quad$ distenduntur] extenduntur (?) $V$ diffunduntur $A T \quad$ eius post membra add. postea subp. $C \quad$ corporis post membra add. postea subp. $M \quad 71$ quomodo] q(ua)n(do) $R \quad$ omnes om. $T \quad$ explanamus $T \quad 72$ et add.s.l. $V \quad$ ab] ex $V$ parte post ea $a d d . T \quad \operatorname{sedecim} A$ sexdecim $V T \quad$ que] qui $R \quad \mathbf{7 4}$ peragunt $T \quad \mathbf{7 5} \mathrm{a} \ldots$ a] in... in $B C M R \quad$ dextra $T \quad$ altera] alia $B R \quad$ a(2)] in $A \quad \mathbf{7 5 / 7 6}$ duobus cathenis $B R$ 
catenis exeunt ipse uene, ab ipsis duobus ossibus que in pectore incipiunt et in humero finiunt.

Iste uene pergunt ad interiora cordis, et omnia forcia uite ibi sunt. Et exinde descendunt due uene maiores per duo latera spinalis et diuiduntur per costas et duos neruos per uniuersa nodora et girant totas costas usque dum ueniant ad costam que dicitur Eua. Et ambo ipsi maiores nerui diuidunt se per uniuersa nodora, duo minores donec ueniunt ad ipsum lumbum. Item alii duo pergunt per medullam spinalis qui descendunt per medullam capitis. Postea diuiduntur in duos neruos. Ipsi sunt qui detrahunt se a duabus partibus cudurilis ad neruum qui dicitur neruus glandualis, usque ad femora. Exinde diuidunt se in quinque uenas usque ad genua et detendunt se ad tibias usque ad talos et diuiduntur per plantas pedis usque ad digitos. Tunc diuidunt se per duas uenas tibie et omne et unum $\mathrm{v}$ uenas. Vnum autem respicit et redit et ascendit usque ad cerebrum capitis et ad uenam que dicitur principalis. Postea descendit et fundit sanguinem in corde ; deinde diuiditur in vi uenas. Due autem circundant pectusculum et exitus illarum ad duo brachia et de brachiis diuidunt se in $\mathrm{v}$ uenas.

Ex ambobus brachiis quatuor detenduntur ad uterum subteriorem subtus umbilicum de duabus partibus. De quatuor uenis istis exeunt adhuc $\mathrm{x}$ uene. Et ascendunt insuper ab intus uterum usque ad gutturem et fundunt in os hominis. Due uero detrahunt sanguinem usque ad dentes. Alie due detrahunt se usque ad molas. Alie due detendunt se usque ad oculos. Due uero campsant se ad aures et alie due detendunt se a cerebro usque ad renes; a renibus autem usque ad duo uirilia. Tunc diuidunt se in uenas paruas ut circumdent duo uirilia atque naturam hominum. Hec sunt diuisiones uenarum atque exitum illarum in corpora. Cuncte natiuitates uene atque ossa et arbores oculi uel ungule medulle atque cerebri et membra que creata sunt habent stillare semen patris. Cuncta natiuitas carnium atque sanguinis et pelles

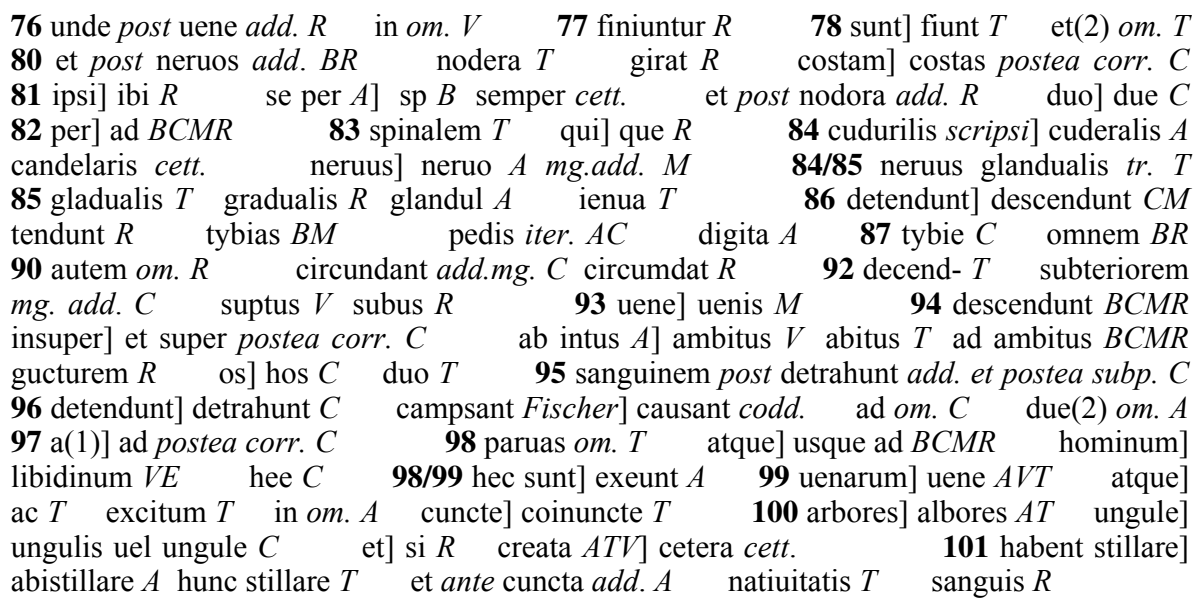


que create sunt agunt semen matris. Spiritus et anelitus, lux et uita, cognoscere bonum et malum a deo uenit, qui est benedictus in secula seculorum amen.

\section{De partibus corporis secundum Asaph. De prima.}

Additque Asaph loquens pro diuisione corporis ad exitus illius interpretans creaturam. Caput est quarta pars corporis, fortitudo cerebri et capitis ; ipse est fons cerebri quod simile est regi sedenti in cubiculo suo, et ipse est rex potentium omnium, corporis fortitudo. Cerebrum calidum est ualde et ipsum est fons scientie. Exinde egreditur flegma que descendit in cerebro ; frigida est nimis uelut nix et mixturat se cum ardore cerebri, quia si flegma uel calor non fuisset, non poterat stare corpus ante ardorem cerebri, eo quod est ualde calidum et siccum, et fortitudo flegme frigida et humida. Proinde preparate ambabus partibus.

\section{De secunda parte corporis.}

Secunda a collo usque ad stomachum. Similiter quarta pars corporis ipsa est qui dicitur uterus superior. Fortitudo eius est epar, quia ipsa est dominatrix uteri. Fortitudo eius ualde calida est et humida et tenera et ipsum fel adheret ei. Ipsum fel frigidum est uelut nix. Idcirco, sicut supra diximus, ita et ibi duo stant quia ordinata est preparatio sedibus eorum, unus aduersus alium, eo quod epar caminus corporis est et dat caliditatem in uisceralibus membris uteri et in calore suo commiscetur cibus.

\section{De tertia parte corporis.}

Tertia pars ab umbilico usque ad spinalem. Ipsa est quarta pars corporis, et uocatur uterus subterior, iuxta renes posita est et forcia renionum hec est. Dexter

102 agunt] a gutta $A \quad \mathbf{1 0 3}$ malum om. $B C M R$ uenit] uelut $B C R$ amen om. $B$ 104 de --- prima] .i. de diuisione corporis $A$ om. $C T$ quattuor post de add. BCMR corporum $C$ corpore $E \quad$ cap. xv postea prima $a d d$. $B E \quad 15$ mg.add. $B R \quad 105$ addit $C$ pro] per $T$ quod $V$ ad om. $B C M R \quad$ exitus] corpus exitus $B \quad 106$ est(1) om. $A$ corporum postea corr. $C \quad 108$ ipse $A \quad 109$ flagma $R$ fleuma $V T$ flema $M$ 110 mixturat scripsi] mixtat codd. flegma uel om. $A$ uel calor om. Fischer 111 stare corpus transp. $R$ corpus $m g$. add. $C \quad$ ardore $A$ calorem $B C M R$ et ante eo add. $T$ est ualde $t r . T 112$ flagme $T$ preparate(-re $V) A T V$ ] preparatio $B C M R \quad$ sunt post preparate add. $A \quad$ ambobus $B \quad$ similiter post partibus add. $T \quad \mathbf{1 1 3}$ de secunda parte corporis om. $T$.ii. pars $A \quad$ corporis] hominis $A M V \quad 16 \mathrm{mg}$. add. $R \quad \mathbf{1 1 4}$ similiter om. $T$ ipsa om. $A \quad$ est om. $R \quad$ ipse est post est add. $A \quad$ ualde $a d d$. postea subp. $C \quad \mathbf{1 1 5}$ est(2) om. $R$ s.l. add. $C \quad$ dominatris $C \quad \mathbf{1 1 6}$ eius] est $C \quad$ est om. $C \quad$ ipsum fel

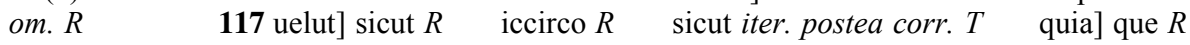
118 preperatio $C \quad$ eo $m g$. add.C 119 uisceribus $B C R T$ et post membris add. $C$ 121 de tertia parte corporis om. $T$ III. $A \quad 17$ mg.add. $R \quad 122$ usque] us $R$ spinale $T \quad$ est quarta pars transp. $R \quad$ est om. $B \quad 123$ subteriorum $M V$ subteriori $T$ iusta $R \quad$ forcia] forciorum $A \quad \mathbf{1 2 3 / 1 2 5}$ dexter --- uelut om. $C$ 
calidus est ualde, eo quod ei calor ab epate uenit. Renio uero sinister frigidus est uelut nix, quia pro ipsa splene non habet talem calorem uelut dexter, quia forcia splenis frigida est uelut gelu. Et ipse est locus colere nigre de subtus renionibus. Ibi est ipsa uesica et congregationes aquarum de ipsa uesica et forcia de ipsa uesica hec est. Est frigida quasi nix et forcia renionis ardet quasi ignis, et si non fuisset calor renionis non poterat corpus stare ante frigiditatem exeuntem de ipsa splene et frigiditatem ipsius uesice, et si non fuisset frigiditas de ipsa uesica non poterat corpus stare ante calorem renionis. Proinde mittunt se inuicem et confirmant corpus.

\section{De quarta parte corporis et conuenientia aut disconuenientia sanguinis.}

Quarta uero pars incipit ab ipso cudurili reuersusque ad plantas pedis tota plena est et non est uacuitas in ea et sanguis ambulat per ipsas uenas et ipsa flegma mixturat se cum ipsa carne, et frigiditas de ipsa flegma inuicem obuiat sibi cum caliditate de ipso sanguine. Et hii duo humores, si steterint equales, tunc corpori sanitatem prestat, et si sanguis superauerit coleram, tunc concitat uulnus aut scabiem. Et si humiliauitur sanguis et superauerit flegma, tunc ueniet frigiditas in lumbis, in coxis, in genua; solent dolere pedes atque tali. Hec est interpretatio corporis secundum partes suas.

\section{De quatuor humoribus, locis et effectibus in homine.}

Locus ipsius colere rubee intus uiscera, subtus cor est. Flegma uero congregationem suam habet in pulmone. Et colera nigra in ipsa splene. Sanguis autem in ipso epate. Isti sunt quatuor humores secundum quatuor elementa mundi, quod est terra, aqua, ignis et uentus. Et sequuntur quatuor circuli annorum, qui ipsi sunt frigidi et calidi, uirides et sicci. Locus intellectus in corde est. Locus sapientie in cerebro capitis. Locus cogitationis et tristitie et afflictionis in renionibus. Locus gaudii et letitie et dulcedinis anime in fontem sanguinis.

$\mathbf{1 2 5}$ uelut om. $C \quad$ splene] plene $T \quad \mathbf{1 2 6}$ plenis $T \quad$ colore $M \quad \mathbf{1 2 7}$ uis(s)ica passim $A$ uexica passim $B M \quad$ uesica(1)] uexica que $C \quad$ et(1)] que $R \quad$ congregans $B$ et ---

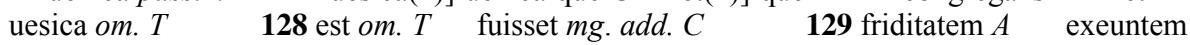
om. $A \quad 129 / 130$ exeuntem --- et om.C 130 non poterat iter. postea subp. $C$ 131 mittunt] nutriunt $B C M R \quad \mathbf{1 3 2}$ de --- sanguinis om. $T$ iiii $A \quad 18$ mg.add. $R$ aut] $\begin{array}{lllll}\text { et } R & 133 \text { uero om. } B & \text { corporis post pars } a d d . C R & \text { cudurile } A & \text { reuersusque] }\end{array}$

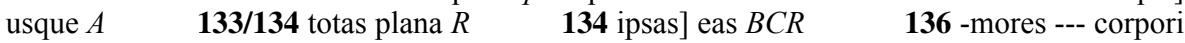
om. $T \quad$ esteterint $R \quad$ corporis $C \quad 137$ prestant $M T \quad$ colera $A \quad 138$ scabiam $A$ humiliatur $C \quad$ uenit $C$ et post ueniet $a d d$. $R \quad \mathbf{1 3 8 / 1 3 9}$ in lumbis in coxis] in coxis et in lumbis $R \quad \mathbf{1 3 9} \mathrm{in}(2)]$ et $B \quad$ coxis $A B C M R \quad$ et post genua $a d d$. BCMRT solent --tali om. $A \quad \mathbf{1 4 1}$ de --- homine om. $A T \quad$ eorum post effectibus add. $B \quad$ xv post homine $a d d$. $C$ xvi mg.add. $B \quad$ capitulum xvi ${ }^{\mathrm{m}}$ add. $E 19$ mg.add. $R \quad 142$ rubee om. $B R$

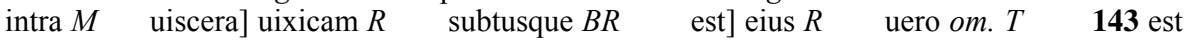
post nigra add. $B C M \quad$ sanguine $R \quad 144$ ipsi $C \quad 145$ secuntur $B C M T$ sequentur $A$ 146 uirides] uiri $T \quad$ intellecti $A$ in corde $A$ ] cordis cett. $\quad 147$ est post capitis $a d d . T$ cogitationis] cognitionis $C$ 
Anime acumen et fortitudinis spiritus et auaritie et amaricationis, suspendunt in colera rubea. Mala cogitatio que contristat uultum, et tribulatio anime et cogitationis spiritus degunt in colera nigra, que potest super corpus multam portare grauitudinem. Et pigritie et humilitatis spiritus degunt in flegmate que potest super corpus multam obliuiscentiam. De multo flegmate si potest super cerebrum tunc descendit flegma super cor et cooperit illud et obliuiscitur omnem causam. Rectitudo scientie et intellectus iuxta rectitatem cerebri in flegmate ; si ipsi equales sunt in commixturationem eorum, etiam scientie et intellectus rectum faciunt quia forcia cerebri ipsa est forcia scientie, et si humiliatur cerebrum ante flegma, humiliatur etiam scientia et intellectus. Qui obseruat inimicitiam et qui uindicat et qui increscit et qui irritat de ante forciam calor uiscerum est.

Caluitia et percaluitia et caput depilatum degunt in colera nigra et omnes dolores qui dant in costis punctam degunt in frigiditatem flegmatis. Per ista intelligimus omnia quia preparatio intellectus in cerebro capitis est. Et lumen a se in oculis et auditus in aures quia sunt qui audiunt et non uident. Et stultitia in lingua est. Verba dulcia et dura in ore sunt, et tristitia in corde est et letitia et dolor in anima, et pietas et iracundia cum locutione a pulmone uenit.

Vox uero a renione exit. Risus in splene. Anelitus in pulmone. Desiderium libidinis et forcia in lumbis est. Lassitudo et fatigium et infirmatio est in pedibus. Labor et opera in manibus sunt.

\section{De partitione anni in quatuor circulis.}

Partitio anni in duodecim mensibus est et in quatuor circulis eius et circulus quarta pars anni est. Tres menses uernum est, et tres estas, et tres menses nouarum frugum et tres uindemie. Condictum uerni frigidum et uiride. Condictum estus

149 fortitudines postea corr. $C \quad \mathbf{1 5 0}$ cogitatio] contristatio $C \quad$ tribulationis $B C M R$ 151 deguntur (?) $C \quad$ potestat $A \quad \mathbf{1 5 1 / 1 5 3}$ multam --- corpus om. $T \quad 152$ et(1) om. $B R$ in om. $A \quad$ potestat $A \quad \mathbf{1 5 3}$ corpus --- super $m g$. add. $C \quad$ multam obliuiscentiam $A$ ] multa obliuiscentia cett. potestat $A \quad \mathbf{1 5 4}$ cohoperit $B C M \quad$ illud] eum $A$

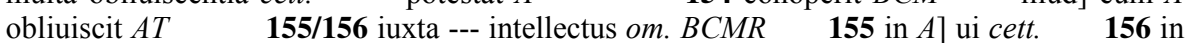
commixta racionem $A \quad 157$ forcia(1)] forciora $T$ est om. $M$ humiliabitur $R$ 158 obseruat] obfuerat $B M R \quad$ qui... qui... qui] que... que... que $R$ uendicat postea

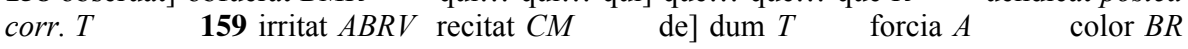
uiscoscerum $C \quad \mathbf{1 6 0 / 1 6 1}$ in colera --- degunt om. $A \quad \mathbf{1 6 0}$ nigra] rubea $B C E M O R T$ 161 costis] cõsas $V \quad$ frigiditate $B C M O \quad \mathbf{1 6 2}$ quia] que $T$ preperatio $C$ intellectus iter. $T$ a se] anime $M R T$ anime a se $A B C \quad 163$ est post oculis add. A qui om. $T$ 164 dura] mollia postea subp. et mg.add. $M$ ore] corde $R \quad 165$ est post anima add. A 166 uero] uera $B R$ om. $C$ hanelitus $B C R \quad 167$ et(1) $B C R V]$ in $A$ laxit- $C$ fatigium $V$ flagitium $C$ fastigium $A B M R T$ et(3) om. $C$ pedibus] pe. $T \quad 169$ de --circulis om. $A T \quad$ xvii post circulis $a d d . B C \quad$ capitulum xviim add. $E \quad 21 \mathrm{mg}$. add. $R$ 170 menses $M R \quad 171$ est post pars add. postea subp. $C$ uernum] neruum $T$ estas $B C$ estus $T$ menses(2) om. $M \quad \mathbf{1 7 2}$ conditum $C R \quad$ condictum nouo(-a-)rum frugum calidum et uiride post uiride $a d d$. $A T$ 
calidum et siccum. Omnis sapor qui dulcis est, ipse est frigidus et uiridis, et omnis sapor amarus calidus et siccus. Omnis sapor uerni continetur a frigiditate sua et siccum est. Ventus auster calidus et humidus. Ventus orientalis calidus et siccus. Ventus liba calidus et uiridis. Ventus aquilo frigidus et siccus. Ventus maris frigidus et uiridis.

\section{De natura cerebri secundum Asaph.}

Aitque Asaph pro anima et forcia corporis atque scientia ipsa est cerebrum capitis, quia dum cerebrum firmatur, tunc et scientia firmatur, et quando cerebrum alleuiatur, scientia minuitur; dum corpus in calorem redditur, cerebrum firmatur ad sanum faciendum uniuersum corpus, quia sicut bona est anima, ita et corpus est. Quia corpus est fons anime et inde est medicina ad cubicula cerebri et capitis. Similiter exinde ad uniuersum corpus descendit, ubi scientia habitat ac prudentia, et dum adimplete fuerint medietates dierum mensium lune tunc crescit cerebrum et implet uniuersa cubicula sua. Tibi enim dicit Asaph : « qui capit medicinam, capit omnem scientiam ».

\section{B. DE YPOCENTAVRO ET QVOMODO CAPTVS EST ET DOCVIT YPOCRATEM.}

Et ambulauerunt parentes cum infantulo suo et infirmatus est et iacuit quasi mortuus et contristati sunt parentes eius et dimiserunt eum in ipsa silua. Et uenit ypocentaurus ad fontem secundum suam consuetudinem et inuenit ibi infantem iacentem quasi mortuum et interrogatus est infans ab ypocentauro et dixit ei : «Vnde hic aduenisti, fili?» Ille autem respondit: "Dum ambularem ego cum meis parentibus per istam siluam et essem circumdatus a graui infirmitate reliquerunt me parentes mei iacentem quasi mortuum. »

Et abiit ypocentaurus ad fontem et inuenit herbam et tetigit infantulum et in ipsa hora infans ad suam memoriam deuenit et ypocentaurus dedit ei herbam bibere

173 sapor $m g$. add. $C \quad$ est(2) om. $T \quad$ uiridus $R \quad 176$ uentus(2) om. $M \quad$ aquilo --siccus $m$ g.add. $M \quad$ frigidus] calidus $C$ aquilo calidus iter. $C \quad \mathbf{1 7 7}$ uiridus $R \quad \mathbf{1 7 8}$ de --- asaph om. $A T$ xviiii post asaph $a d d$. $B$ xviii $a d d . C$ ad scientiam et sanitatem xviii (capitulum xviiim $E$ ) add. $B C E \quad 21 \mathrm{mg} . a d d . R \quad 179$ cerebrum] cereber $A$ 180 tunc --- firmatur om. $T$ formatur $C \quad 181$ et post alleuiatur $a d d$. $A C$ colorem $C$ tunc post redditur add. $R \quad$ firmatum $C \quad \mathbf{1 8 2}$ bona om. $R$ corpus(2) mg. add. $C$ 183 quia corpus est om. $V \quad$ ipse post corpus add. $A \quad$ inde] ipsa $C \quad \mathbf{1 8 4}$ descendit post exinde colloc. $A \quad$ descendat $M \quad$ ac] et $R \quad$ et $V$ ] est $A B C M R T \quad 185$ crescit om. $R$ 186 tibi] inde $R \quad$ medicinam] medicina in $C \quad 188$ de --- ypocratem om. $A$ pocentauro $R$ dedocuit $R \quad$ xix post ypocratem add. BC capitulum xixm add. $B 22$

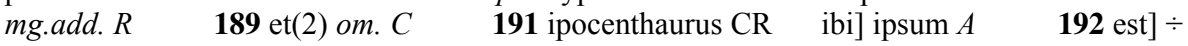
$A$ ipoc- $C R \quad 193$ cum $R$ ambularem ego transp. $R \quad 194$ et essem circumdatus] circumdatus sum $A$ graui] forte $A$ et post infirmitate $a d d$. A 195 iacentem om. A 196 et abiit --- inuenit iter. $T$ ipsam post ad add. $C \quad$ 196/197 et(2) --- infans] et ipse infans in ipsa hora $A \quad 197$ omnem post ad add. A ipse post et $a d d$. A 
et sanatus est puer in illa hora. Et dixit ei «uade et ambula», et dum ambularet inuenit parentes suos et interrogatus est ab ipsis quis eum sanasset, et duxerunt eum ad Ypocratem et dixit ei : « dic michi quis te sanum fecit. » Et dixit ei puer : «dum iacerem per infirmitatem, sic uenit ypocentaurus ad me, qui habebat caput et manus sicut homo et alia similitudo sicut caballus; ambulauit ad fontem et dedit michi herbam bibere et statim in ipsa hora factus sum sanus. »

Sic ambulauit Ypocras cum retibus ad ipsam siluam ut eum posset comprehendere et euacuauit illum fontem et posuit ibi ollam plenam uino et bibit ypocentaurus et inebriatus est et comprehensus est ypocentaurus in rete apud Ypocraten, et interrogatus est ypocentaurus ab Ypocrate de omni arte medicine, et sic factus est Ypocras medicus in omnem terram tempore Diocletiani imperatoris et Vlpii proconsulis. Et inuenit Ypocras quomodo fuit ypocentaurus eruditus in arte medicinali et sic tradita est medicina Ypocrati expertissimo medico. In diebus illis sic ypocentaurus inuentus est. Nam tempore Diocletiani imperatoris inuentus est ypocentaurus xixmo Kł. Ottobr. et comprehensus est ab Ypocrate.

\section{De uenis neruis ossibus humoribus hominis secundum Galienum.}

Quid ad artem medicine pertinet Galienus interrogans magistrum suum Ypocratem dixit : «Trade nobis saltem quanta nomina sunt pigmentorum. » Ypocras respondit: «Genera sunt pigmentorum quatuor unde omnes medicamentorum confectiones fiunt. » Galienus interrogauit magistrum suum Ypocratem « Vnde sunt

198 ipse post est $a d d$. $A \quad$ in] ab $B R \quad$ parte post illa add. postea subp. $C \quad$ uade] uide $T$ 199 et(1) om. $R \quad$ ipsis] eis $C \quad$ sanasset] sanum fecisset $B C$ at sanos $m g . B$ sanum fecisset ał. sanasset $M R \quad$ eum om. $C \quad \mathbf{2 0 0}$ ypocrates $A \quad$ ypocrates post ei(1) add. $A$ michi om. $M$ te sanum transp. $A B C R \quad 201$ per] pre $C$ ab $A \quad$ infirmitate $A$ ad me] nomine $A \quad 203$ herbam bibere transp. $C \quad$ in om. $T$ factum $C \quad 204$ sic] tunc $B C M R m g$. al. sic $B \quad$ ipsam] eandem $B C M$ eundem $R$ dictam $V T$ eum] eam $M$ om. $R$

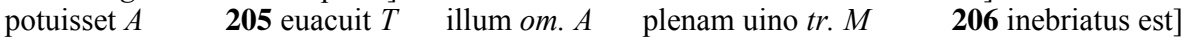
$\begin{array}{lllll}\text { inebriauit se } B R & \mathbf{2 0 7} \text { ypocratem } R & \text { est om. } C & \text { medecine } R & \mathbf{2 0 8} \text { sic] ita } a d d .\end{array}$ postea subp. $C \quad$ diocliciani $A$ dioclesciani postea corr. $T \quad 209$ uenit $R$ fuit om. $T$

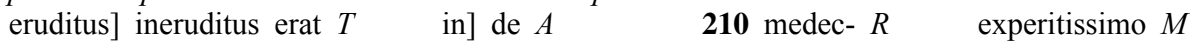
experimentissimo $A \quad \mathbf{2 1 1}$ sic] et $R \quad$ ypocentaurus inuentus est transp. $A$ in post nam add. $A$ nam tempore diocletiani imperatoris inuentus est post inuentus est add. post. subp. $C \quad$ imperatoris om. $T \quad 212$ ypocentaurus om. $A \quad$ xix $B C M \quad 19 R$ xio $T \quad \mathrm{k}$ $A C V]$ kłis $R$ kłas $B \quad$ octubris $A C \quad$ et om. $C \quad$ est $] \div A \quad \mathbf{2 1 3}$ de --- galienum om. $A T$ et post neruis add. BCR et post ossibus add. $C R$ ac add. $B \quad 23$ mg.add. $R$ capitulum xix ${ }^{\mathrm{m}}$ post galienum $a d d . E$ xx.add. $B C \quad 23$ add. $R \quad \mathbf{2 1 4}$ quid] que $A$ medecine $R \quad$ pertinent $A \quad$ sciencia post pertinent $a d d$. $A \quad$ interrogans $B V$ ] interrogauit $A$ interrogat $C M$ (post.corr.)R 215 et post ypocratem add. $A \quad$ saltem] uel $A$ pygm- $C$ pigmentarum $A M \quad$ sunt pigmentorum transp. $A B C R T \quad \mathbf{2 1 6}$ respondet postea corr. $M$ sunt post quatuor colloc. $M \quad 217$ item post fiunt add. BCMR interrogat BM (postea corr. M) -craten passim A uel post ypocratem add. A $\quad \mathbf{2 1 7 / 2 1 8}$ sunt creata transp. $A$ 
creata ista nomina pigmentorum?» Ypocras dixit ad Galienum : « Nouit dominus, quia dominus de terra creauit medicinam.» Galienus dixit ad Ypocratem: «Piissime magister, trade nobis unde fiunt ista nomina pigmentorum. » Ypocras dixit: "Fiunt de terra, de herba, de arboribus, et de gummis. De istis quatuor generibus fiunt omnia pigmenta que ad artem medicinalem pertinent. »

Galienus interrogauit magistrum suum Ypocratem: «Trade nobis quotas uenas habet homo in toto corpore suo.» Ypocras dixit ad Galienum : «Sunt autem in homine uene trecente sexaginta quinque. Nam quatuor sunt uene, due spiritales et due sanguinales. » Galienus interrogauit magistrum suum Ypocratem : "Trade nobis quote uene sunt unde sanguinem per rationem extrahere debeamus. » Ypocras dixit : "Viginti uene sunt in toto corpore hominis quas tangere debemus cum flebothomo ad extrahendum humorem. » Quando bona est flebotomia? Ypocras dixit : «Bona est flebothomia cata horam primam uel secundam aut tertiam, quia colera et flegma et melanconia et sanguis dominantur. »

Ypocras et Alexander et Galienus dixerunt quo tempore catarticum accipiendum est. Bonum est mense aprilis et maii accipere catarticum, sed in illis mensibus qui habent « $\mathrm{r} »$ uetamus sumi.

Ypocras dixit ad Galienum : «quatuor sunt tempora et quatuor sunt anguli, quatuor sunt humores in homine; est colera rubea et flegma et sanguis et melanconia; sed de istis humoribus generantur septuaginta quinque humores.» Galienus dixit ad Ypocratem : «Trade nobis quota ossa habet homo in toto corpore

218 nouit] uiuit $A$

219 dominus om. $M$ (postea $m g$. add.) $R$

unde fiunt pigmenta post medicinam add. BCMR dixit] dre (?) $B \quad \mathbf{2 2 0}$ magister] pater $B C R \quad$ uel post nobis add $A \quad 221$ et post terra add. $A$ herbis $A$ etiam post herba add. $A$ gummibus $R \quad \mathbf{2 2 2} \operatorname{arcem} T \quad$ medicinalem] medicine $A$ medinalem $C$ medicinalem scientie $V$ medicinalis scientie $T$ item post pertinent add. BCMR $\mathbf{2 2 3}$ uel post nobis add. $A \quad \operatorname{cotas} R$ quantas $A \quad \mathbf{2 2 4}$ toto om. $R$ suo om. $A \quad \mathbf{2 2 4 / 2 2 5}$ in homine uene transp. $A \quad \mathbf{2 2 5}$ uene $m g . a d d . M$ posite post uene add. $A$ trecenta $A$ et post sexaginta add. $A \quad$ sunt s.l. add. $C$ et om. $R \quad \mathbf{2 2 6}$ item post sanguinales add. $B C M R \quad 227$ quante $A$ subtrahere $A$ debemus $C R$ que uene sunt in homine que tangi debent post debeamus add. BCMR s.l.add. $V \quad$ ypocras dixit om. $A \quad \mathbf{2 2 8}$ toto

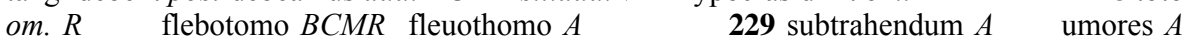
quando --- flebotomia mg. add. $V$ om. $A$ bonum $A \quad \mathbf{2 3 0}$ flebotomia $B C R$ fleuotomum $A$ cata] circa $A B C M R \quad$ aut] uel $B C M R \quad 231$ et(1)] uel $C$ melanconia scripsi] malenconia $T$ melanconicus $A$ melancolía cett. et sanguis om. $A$ et(2) om. $C$ quo tempore catarticum accipiendum est post dominantur $m g$.add. $V \quad \mathbf{2 3 2}$ et (1) om. BOER

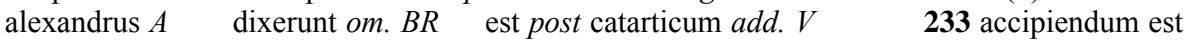
transp. $A B T \quad$ mai $A \quad$ accipe $R \quad 234$ mensibus] mensis $C \quad$ que $R$ sumi scripsi] summi $A$ sompnum cett. 235 sunt tempora] sunt sut $B \quad \operatorname{sunt}(2)$ om. $A$ et om. $C R$ anguli $A$ ] angłi (ãgłi $B$ ) $B C M R T$ angeli $V \quad \mathbf{2 3 6}$ homines $A$ rubera $B \quad \mathbf{2 3 7}$ melanconia scripsi] malancolia $R$ melenc- $T$ melancolicus $A$ melancolia cett. de] in $B R$ om. $C$ $\operatorname{lxxv} M 65 R \quad$ et post septuaginta $a d d$. $A \quad$ adixit $C \quad \mathbf{2 3 8}$ ypocras dixit ad galienum $A$ ypocraten dixit ad galienus post dixit ad interpun. $V$ quanta $A$ toto mg.add. $M$ 
suo ». Ypocras dixit : «Centum nonaginta quinque et nerui sunt in homine centum numero, pelle uero induti sunt, sicut homo corio indutus est. Sanguis autem hominum uirtus anime est. Lingua autem ut loquatur. Oculi ad uidendum. Manus ad operandum. Pedes ad ambulandum. Caput ad bonum faciendum et ad bonitatem et castitatem et munditiam conseruandam. Sancte autem et caste uiuet qui secundum me et artem meam conformabitur. »

C. Asclepius puer factus Apollinis et ex Coronida filia Flabie, cironicentauro traditus est ad medicine scientiam comprehendendam. Postea egrior factus silue et medicine disciplinam interrogauerunt istum doctorem et Asclepius et Apollo scrutati sunt rationabiliter artem medicine et Ypocras tradidit explanationem artis medicine.

\section{1. DE ASSIMILATIONE ELEMENTORVM.}

Quomodo factus est mundus, taliter de istis quatuor causis, igne, aqua, puluere et uento. Ignis assimilatus est sicut peccatum qui concitat corpus hominum ad faciendam malignitatem. Aqua assimilata est ad elemosinam, quia sicut aqua extinguit ignem, ita elemosima extinguit peccatum. Puluis assimilatus est terre, quia de humore terre factus est homo. Ventus assimilatus est aeri, quia de aere conformatus est spiritus et corpus humanum. Sicut ait Dauid psalmographus: «Exibit spiritus eius et reuertetur in terram suam. In illa die peribunt omnes cogitationes eorum.» Homo assimilatus est sicut flos campi, sicut scriptum est in ystoria. Mane germinat et floret ad uesperam marcescit et siccat, tamquam si numquam fuisset in seculo natus. Sicut de quatuor causis constat corpus humanum, ita mundus similiter de quatuor causis constat.

Corpus humanum in quatuor partibus diuiditur : caput, pectus, umbilicum et

239 et post nonaginta $a d d$. $A \quad$ nerui] neruora $A \quad \mathbf{2 4 0}$ numero om. $A$ pellem $A$ induti --indutus est] in homines sicut corium indutum est $A$ homo post indutus est add. postea subp. $C \quad \mathbf{2 4 1}$ hominis BCMR 242 et ad om. $A \quad \mathbf{2 4 3}$ faciendam post castitatem add. $A$ inmunditiam $C$ uiuet qui] conseruet $A \quad \mathbf{2 4 4}$ me] meum $A$ $\begin{array}{lll}\text { conlaudabitur } A & \text { de explanatione medicine post conformabitur add. BCEMOR } 24\end{array}$

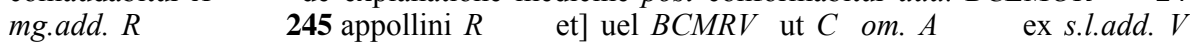
coronitida $T \quad$ cyroni- $B C \quad 246$ agrior $A \quad \mathbf{2 4 6 / 2 4 7}$ et medicine transp. $A$ 247 et ante disciplinam $a d d$. $T$ inuenerunt $A$ istos doctores $A$ et om. $A$ uel esculapius post asclepius $a d d$. $R \quad$ appolo $R$ Apollonius $A \quad \operatorname{et}(2)]$ uel $B \quad$ scrutauerunt $A$ 248 ars $A$ et --- medicine om. $T$ et] sic add. BCRMV ypocrates $A C$ (postea corr. $C) \quad$ explanact- $R \quad \mathbf{2 4 9}$ de assimilatione elementorum] om. $A$ quomodo factus est mundus $T$ et partibus \& sensibus \& quatuor humoribus \& de locis (rolocis $R$ ) \& effectibus eorum in homine $a d d$. $B R$ iiii $^{\text {or }}$ post assimilatione $a d d$. $B C R \quad 21$ add.mg. $B 25 \mathrm{mg}$. add. $R$ 250 est] et $R \quad$ igni $B R \quad \mathbf{2 5 2}$ elimos- $R \quad \mathbf{2 5 3 / 2 5 4}$ terre --- est om. $B$ 255 humanum om. $C$ ait] autem $T$ psalmigraphus $B$ salmigrafus $R$ $\mathbf{2 5 6}$ perhibunt $C \quad$ omnes s.l. $T \quad \mathbf{2 5 7}$ est(1) om. $R \quad \mathbf{2 5 8}$ istoria $C$ hist- $M$ marcessit $M R \quad 259$ nunquam fuisset transp. $R \quad \mathbf{2 6 0 / 2 6 1}$ ita --- humanum $m g$. add. $C \quad 260$ ita om. $A \quad \mathbf{2 6 1}$ et post pectus $a d d$. A umilicum $R$ 
uesicam. Caput autem est quarta pars corporis et est fons cerebri, quasi rex qui sedet in sede sua. Ipsum enim regnificat super totum corpus hominis et forcia medullarum que calida est. Et exinde procedit scientia et exitus flegme. Exinde procedit quod ipsa frigida est, et frigidum continet, sicut calorem cerebrum; quod si calor cerebrum non fuisset nunquam poterat euadere corpus humanum de frigiditate flegme. Et si frigiditas flegme non fuisset non poterat euadere corpus humanum propter calorem cerebri. Quoniam cerebrum calidum et aridum est, et flegma frigidum et humidum. Propterea creata sunt unum contra aliud.

Quinque talenta sunt in natura humana : uisus, auditus, odoratus, gustus et tactus, et in quatuor partes diuiditur corpus humanum: caput, stomachum, uentrem adque uesicam. Caput imperium est; stomachus regnum est; uenter gurgus est; uesica autem mercenaria. Si caput doluerit, totum corpus perturbatum est.

262 ipsa post autem add. $A$ autem om. $R \quad$ est quarta pars transp. BCMR ipsa post est(2) add. $A \quad$ cerebrorum $A \quad \mathbf{2 6 2 / 2 6 3}$ sedet in sede sua transp. $A$ et post sedet add. $A$ 263 sede sua $A$ ] sedem suam cett. $\quad$ ipse $A$ enim om. $A$ forcia] forma $T$ medula- $R$

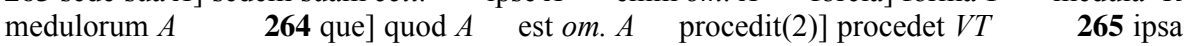
om. $C$ et scripsi] sicut codd. calorem scripsi] calor codd. quod] quia $B C M R$ $\mathbf{2 6 6}$ cerebrum $T$ ] cerebri cett. nunquam] non $R \quad$ poterat] potuerat $A \quad \mathbf{2 6 7}$ euadere corpus humanum transp. $C \quad \mathbf{2 6 8 / 2 6 9}$ aridum --- frigidum et om. $T \quad \mathbf{2 6 9}$ cerebrum post frigidum $m g$. add. $C \quad$ frigida... humida $A$ est post humidum add. $A T$ create $A$

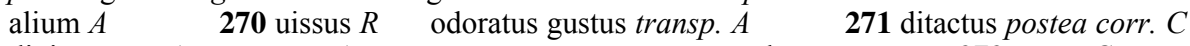
diuisum est $A \quad$ uenter $A \quad$ caput atque uentrem stomachum $M \quad \mathbf{2 7 2}$ atque $C$ ac $R$ 273 autem om. $A$ mercenna- $B C M$ mercandaria $R$ est post mercenaria add. $A B C$ doluit $R \quad$ est om. $R$ 